\title{
Editorial
}

\section{Possible uses of gene therapy in reducing coronary restenosis}

Gene transfer has been carried out in more than 700 patients as part of over 200 registered protocols in the United States and Europe since 1989. Although success rates vary and many efficacy and safety issues remain unsolved, the potential of this alternative therapeutic method is well accepted. The lack of success of many pharmaceutical agents in reducing restenosis rates after angioplasty shown in dozens of clinical trials has encouraged the development of gene therapy as a further treatment. The introduction of special catheters for local drug delivery allows high efficacy combined with maximum safety.

\section{Pathophysiology of restenosis}

Four overlapping stages occur in the development of restenosis as described in animal models. The inflammatory and thrombotic phases begin at the time of injury and are maximal hours later. These are followed by a proliferative phase, with the highest division activity of smooth muscle cells about seven days after injury. Finally, there is matrix formation from one week onwards. ${ }^{1}$ As part of these processes, extracellular growth factors bind to cell surface receptors and can initiate the cascade required for signal transduction that leads eventually to cell division. Protooncogenes in the normal growth-regulatory pathway are stimulated by these factors, are transiently switched on, and, together with other cell cycle related genes, regulate cell division. Cell proliferation in restenosis may also result from a reduction in inhibitory cell cycle controlling factors.

\section{Methods of gene transfer}

PHYSICAL MEANS

Local drug delivery devices have been developed to achieve increased regional concentrations of transferred agents, including genes. The arterial wall is easily accessible with a percutaneous transluminal approach and this facilitates local therapy. If suitable drug application is performed at the time of angioplasty, no further intervention may be necessary.

\section{MOLECULAR MEANS}

The goal of gene modification in restenosis is to produce a transient and localised specific effect on certain cells and thus reduce cell proliferation or matrix formation, induce angiogenesis, or influence remodelling. Genetic material may be delivered as naked DNA, complexed with transfection agents such as liposomes or by viral infection usingfor example, replication deficient retroviruses or adenoviruses. Transfection describes the transient delivery of DNA into the cell, where the genetic material has a relatively short lived effect. Antisense oligonucleotides for inhibition of transactivation of cell cycle stimulators (decoy sequences) and protein synthesis can be effective in both the nucleus and cytoplasm; in contrast, plasmid DNA needs to enter the nucleus to allow gene expression (fig 1).

Spontaneous integration of plasmid DNA into the genome is rare. Much more efficient is infection by viral vectors, including recombinant retroviral or adenoviral particles. Retroviral vectors allow stable integration of genes selectively into proliferating cells at low efficiencies, whereas adenoviral transfer typically leads to transient and non-selective transfection with higher efficiency.

In principle, therapy in vivo can be aimed at inhibiting normally occurring growth stimulators or augmenting natural growth inhibitors. Alternatively, therapy can intend to kill cells with the introduction of cytotoxins. In general, the lower in the signal transduction cascade cell regulation is influenced, the more predictable are the effects achieved, therefore minimising adverse events (fig 2).

\section{Inhibition of growth stimulators}

Most growth factors are multifunctional, therefore unwanted effects may occur following inhibition. Fibroblast growth factor (FGF) is a potent mitogen during the first phase of restenosis, but it can also stimulate angioneogenesis ${ }^{2}$ and may be beneficial in reducing ischaemia if total occlusion of arteries is a possibility. Hence the price of reduction in smooth muscle cell growth might be reduced angioneogenesis. Transforming growth factor (TGF) $\beta 1$ expression is increased in restenosis. ${ }^{3}$ Reduction of expression results in a substantial decrease in extracellular matrix production; however, the effect on cell proliferation is concentration dependent. At high concentrations, TGF- $\beta 1$ is a natural growth inhibitor, while at low concentrations it stimulates cell growth; thus reduction of TGF- $\beta 1$ may increase intimal proliferation. The effectiveness of growth factor inhibition may be reduced by concurrent enhancement of other factors and by role changing under varying conditions. Inhibition of gene expression has mostly been undertaken using antisense oligonucleotides. Although the drug regulatory authorities view therapy with these differently from the transfer of whole genes, transfer of short lengths of DNA is recognised as gene transfer by most researchers. Despite some beneficial results in animal models, mostly targeting proto-oncogenes, the therapeutic usefulness and specificity of antisense oligonucleotides is controversial. Oligonucleotides are only short sequences and thus there is a relatively high chance that these sequences might bind to

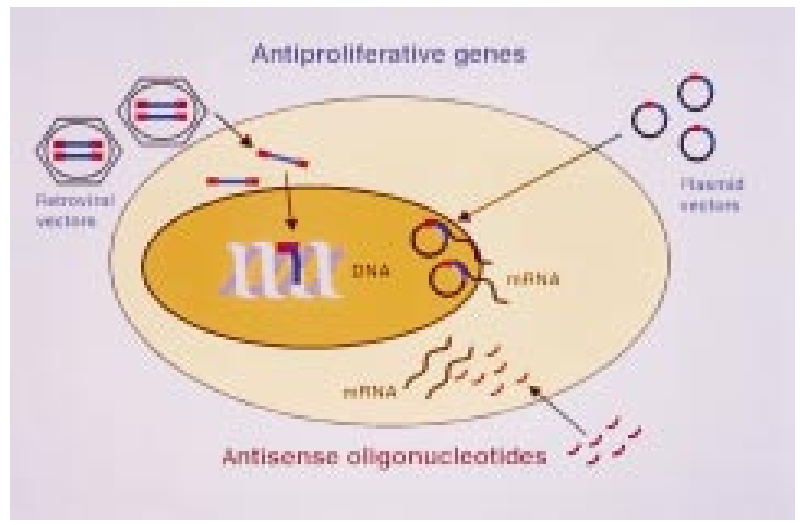

Figure 1 Representation of a cell showing three possible approaches to influence protein formation and thus reduce cell proliferation using gene therapy. Augmentation of antiproliferative genes can be by retroviral integration of the therapeutic gene into native DNA or by transfection with expression plasmid vectors. Inhibition of naturally occurring growth stimulators can be through antisense oligonucleotides. 
other non-target genes. Further, incomplete matching of the nucleic acid sequence can also produce undesirable phenotypes by suppressing an unintended gene. Oligonucleotides can also bind to intracellular proteins, altering their function, or they may have a direct toxic activity on the proteins themselves. ${ }^{4} \mathrm{~A}$ clinical trial testing the local transfer of c-myc oligonucleotides following angioplasty for the prevention of restenosis is currently underway.

\section{Augmentation of growth inhibition}

Suitable vectors can be engineered to contain the protein encoding sequences of genes for growth inhibition and angiogenesis. Inducible promoters allow some control over the length and intensity of the expression period. A number of investigations testing the augmentation of cell cycle inhibitors or differentiation genes promoting the phenotype change of smooth muscle cells to the quiescent state in vivo have been undertaken. One such protein is the retinoblastoma protein that prevents the cell from moving from $\mathrm{G}_{1}$ further through the cell cycle. ${ }^{6}$ In both rat and pig arteries, local in vivo administration of a nonphosphorylatable active form of the retinoblastoma gene product reduced neointima. ${ }^{7}$ The passage through the cell cycle is also regulated by stimulating enzymes known as cyclin dependent kinases (Cdks), active when associated with cyclins that are expressed with a certain periodicity during the cell cycle. Proteins such as p15, p16, p18, p21, and p27 are inhibitors of the Cdks and thus prevent entry into the subsequent phase of the cell cycle. The protein p21, also known as senescent cell derived inhibitor 1 inhibits the cell cycle and has particular potential for use in gene therapy. It inhibits $\mathrm{Cdk}$-cyclin complex mediated phos- phorylation of the retinoblastoma protein. Adenovirus mediated overexpression of $\mathrm{p} 21$ has successfully inhibited neointima formation in rat carotid ${ }^{6}$ and pig iliofemoral arteries. ${ }^{8}$ Another important group of growth inhibitors are homeobox gene products, associated with the control of cell differentiation. Initially, these seemed to be mainly relevant to embryogenesis, needed to establish diverse pathways of morphogenesis. It has recently been shown that homeobox genes can also determine cellular phenotype later in life. In the early stages after arterial injury, the tissue specific homeobox gene gax (growth arrest homeobox) is rapidly downregulated during the proliferative response. ${ }^{9}$ It may prove to be a superior target for gene therapy compared with-for example, p21, as it has defined tissue specificity.

\section{Angiogenesis}

The administration of the gene for vascular endothelial growth factor (VEGF) might provide a combination of both growth reduction and angiogenesis, both of which might be useful for reducing restenosis. Even low transfection efficiencies may lead to physiological levels of the biologically active protein because it may be secreted by transfected endothelial cells and thus act in a paracrine fashion. Increased proliferation of endothelial cells induced by VEGF allows rapid repair of the endothelium and thus potentially results in reduced neointimal hyperplasia. At the same time, new collateral vessels may be formed, increasing blood perfusion in ischaemic areas. Three clinical gene therapy trials for the treatment of vascular disease have been approved by the Recombinant DNA Advisory Committee of the National Institutes of Health in the

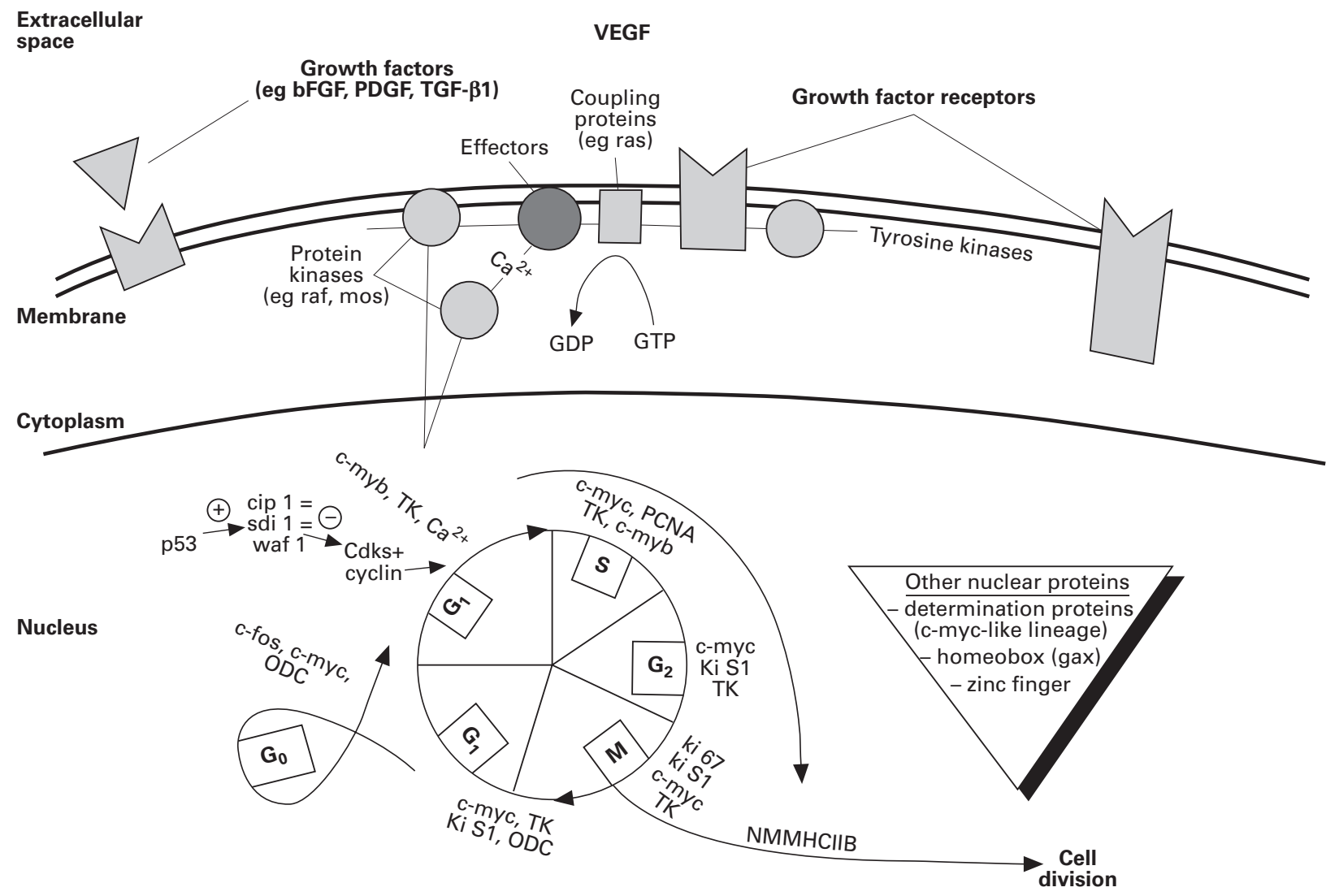

Figure 2 Extracellular, membrane and intracellular control points for cell division, showing some of the protein interactions where gene therapy might act. $b F G F$, basic fibroblast growth factor; Cdks, cyclin dependent kinases; gax, growth arrest homeobox; $M$, mitosis; PCNA, peripheral cell nuclear antigen; RB, retinoblastoma protein; $S, D N A$ synthesis; sdi 1 , senescent cell derived inhibitor $1 ;$ TK, thymidine kinase; TGF- $\beta 1$, transforming growth factor $\beta 1$; VEGF, vascular endothelial derived growth factor; PDGF, platelet derived growth factor; cip 1, Cdk interacting protein; ODC, ornithin olecarboxylase; NMMHC II B, non-muscle myosin heavy chain II B; waf 1 , wild-type $p 53$ activated fragment. 
United States. Two of these aim to improve ischaemia in severe peripheral artery disease. Local delivery of the VEGF gene is via a hydrogel coated balloon catheter or by subcutaneous delivery of VEGF DNA. Twenty patients have so far been included and, at high dosages, promotion of angiogenesis with improvement of blood perfusion has been demonstrated. ${ }^{10} \mathrm{~A}$ second aim is to prevent restenosis following percutaneous transluminal angioplasty of peripheral arteries, again via a catheter based approach. To date, 10 patients have been included and no restenosis event has been documented so far (Isner, 1997, personal communication).

\section{Introduction of foreign genes}

One way of stopping myointimal hyperplasia is selectively to eliminate cells proliferating after vascular injury. One such method uses the herpes simplex virus thymidine kinase gene that encodes for an enzyme that allows phosphorylation and thus incorporation of nucleoside analogues into newly synthesised DNA, which will subsequently lead to blockage of DNA polymerase and premature DNA chain termination. Toxic metabolites may enter neighbouring cells via gap junctions thus leading to a so called bystander effect. This drug induced suicide gene has been successfully introduced into arteries by adenoviral transfer. ${ }^{11}$ The intima:media ratio was reduced following subsequent systemic therapy with intravenous ganciclovir, which turned on the suicide. Importantly, cellular protein release following cell lysis may contribute to the restenosis process; therefore, strategies aimed at slowing or halting cell division with minimal or no cell damage are preferable.

A group of genes that utilises this approach in the field of gene therapy encode for insect antibiotics. One example is cecropin that exists in several precursor configurations. Premature cecropin can influence mitochondrial functions by uncoupling oxidative phosphorylation, inhibiting respiration, and interfering with protein importation. In addition, it may affect transcriptional control. In contrast, its mature form, which may be secreted, causes pore formation in cellular membranes through its bipolar hydrophilic-lipophilic structure, leading to cell death. Whereas mature cecropin kills cells, premature cecropin leads only to reduced cell proliferation and possibly matrix secretion, thus stimulating the use of its gene in restenosis. We have shown that even low transfection efficiencies may lead to a significant reduction of neointima following vascular injury when the cecropin gene is injected into the adventitia of a porcine model. ${ }^{12}$

\section{Conclusion}

Past and ongoing gene transfer studies that aim at reduction of coronary restenosis indicate that the future may lie in the combination of an ideal gene with an optimal local drug delivery device. Naked DNA may be used if gene products are secreted, as low transfection efficiencies seem to be sufficient. However, if gene products remain intracellular, potent vectors including recombinant viruses may be needed. Augmentation of beneficial genes appears to be the method of choice because no suitable inhibitory (blocking) strategy is available. Modulation of gene expression is more promising downstream than upstream, potentially avoiding as many unwanted effects as possible. Suitable targets may include cell cycle inhibitors, homeobox factors, angiogenic VEGF or certain cyotoxic foreign genes that can also slow down physiological cellular functions instead of killing cells.

$\mathrm{SN}$ is supported by grants from the Deutsche Forschungsgemeinschaft and the Bundesministerium für Bildung, Forschung, Wissenschaft und Technologie. The author thanks Dr Tanya Y Huehns for help in preparing this manuscript.

S NIKOL

Medical Department I,

Klinikum Großhadern,

Ludwig-Maximilians University,

Marchioninistr 15,

D-81377 Munich, Germany

1 Nikol S, Huehns TY, Höfling B. Molecular biology and post-angioplasty restenosis. Atherosclerosis 1996;123:17-31.

2 Nabel EG, Yang ZY, Plautz G, Forough R, Zhan X, Handenschild CC, et al. Recombinant fibroblast growth factor-1 promotes intimal hyperplasia and angiogenesis in arteries in vivo. Nature 1993;362:844-6.

3 Nikol S, Isner JM, Pickering JG, Kearney M, Leclerc G, Weir L. Expression of transforming growth factor- $\beta 1$ is increased in human vascular restenosis lesions. F Clin Invest 1992;90:1582-92.

4 Burgess TL, Fisher EF, Ross SL, Bready JV, Qian Y, Bayewitch LA, et al. The antiproliferative activity of c-myb and c-myc antisense oligonucleotides in smooth muscle cells is caused by a nonantisense mechanism. Proc Natl Acad Sci USA 1995;92:4051-5.

5 Villa AE, Guzman LA, Poptic EJ, Labhasetwar V, D'Souza S, Farrell CL, et al. Effects of antisense c-myb oligonucleotides on vascular smooth muscle cell proliferation and response to vessel wall injury. Circ Res 1995;76:50513.

6 Chang MW, Barr E, Lu MM, Leiden JM. Adenovirus-mediated overexpression of the cyclin/cyclin-dependent kinase inhibitor, p21 inhibits vascular smooth muscle cell proliferation and neointima formation in the rat carotid artery model of balloon angioplasty. F Clin Invest 1995;96:22608 .

7 Chang MW, Barr E, Seltzer J, Jiang Y, Nabel GJ, Nabel EG, et al. Cytostatic gene therapy for vascular proliferative disorders with constitutively active form of the retinoblastoma gene product. Science 1995;267:518-22

8 Yang Z-Y, Simari RD, Perkins ND, San H, Gordons D, Nabel GJ, et al. Role of the p21 cyclin-dependent kinase inhibitor in limiting intimal cell proliferation in response to arterial injury. Proc Natl Acad Sci USA 1996;93:7905-10.

9 Weir L, Chen D, Pastore C, Isner JM, Walsh K. Expression of gax, a growth arrest homeobox gene, is rapidly down-regulated in the rat carotid artery during the proliferative response to balloon injury. $f$ Biol Chem during the prolif

10 Isner JM, Pieczek A, Schainfeld R, Blair R, Haley L, Asahara T, et al. Clinical evidence of angiogenesis after arterial gene transfer of phVEGF165 in patient with ischaemic limb. Lancet 1996;348:370-4

11 Ohno T, Gordon D, San H, Pompili VJ, Imperiale, MJ, et al. Gene therapy for vascular smooth muscle cell proliferation after arterial injury. Science 1994;265:781-4.

12 Nikol S, Huehns TY, Schrittenloher P-K, Mrochen S, Traxler H, Günzburg WH, Höfling B. Inhibition of neointimal hyperplasia following local drug delivery of the gene of an amphipathic peptide with the needle injection catheter [abstract]. Proc Eur Atheroscler Soc 1996:29. 